\title{
Eficácia da eletroestimulação muscular expiratória na tosse de pacientes após acidente vascular encefálico
}

\author{
Effectiveness of electrical stimulation in expiratory muscle on cough of patients after stroke
}

André Luís Ferreira de Meireles', Louisiana Carolina Ferreira de Meireles², José Carlos Eugênio da Silva Queiroz $^{3}$, Rafael Miranda Tassitano ${ }^{4}$, Fernanda de Oliveira Soares ${ }^{5}$, Adriana Siqueira de Oliveira ${ }^{6}$

\begin{abstract}
RESUMO I Indivíduos com sequelas de acidente vascular encefálico (AVE) apresentam como complicação comum a hipotonia de tronco com consequente fraqueza da sua musculatura, em especial, o reto abdominal que é o principal músculo acionado para a tosse. As correntes elétricas têm sido amplamente utilizadas para recrutamento de fibras musculares nas mais diversas patologias. O presente estudo teve como objetivo avaliar a eficácia da eletroestimulação transcutânea de média frequência (ETMF) na força muscular expiratória e tosse de pacientes com sequela de AVE. Trata-se de um estudo quase-experimental (antes e depois) onde foram selecionados 11 indivíduos pós-AVE entre 40 a 65 anos, ambos os sexos e estáveis hemodinamicamente. Os pacientes foram avaliados quanto a parâmetros clínicos e pneumofuncionais (ventilometria, pico de fluxo expiratório (PFE) e manovacuometria) e submetidos ao protocolo, que constava de ETMF no músculo reto abdominal com o aparelho de corrente Russa, frequência portadora de $2.500 \mathrm{~Hz}$ modulada a $40 \mathrm{~Hz}$, durante 15 minutos, por 15 sessões. Na avaliação inicial observou-se diminuição da força muscular inspiratória e expiratória (Pimáx; Pemáx) e do PFE quando comparados aos valores de normalidade preditos na literatura. Após a ETMF houve incremento da Pimáx e Pemáx sem significância estatística $(p=0,18$ e $p=0,29)$ já 0 PFE teve um acréscimo de 283,73 L/minuto para 347,27 L/ minuto $(p=0,03)$. Pode-se observar que a ETMF foi eficaz no incremento dos parâmetros avaliados, com o PFE sendo o de maior impacto e significância estatística, no entanto,
\end{abstract}

ABSTRACT I Individuals with sequelae of stroke present as a common complication hypotonia and weakness of trunk muscles, in special the rectus abdominal muscle which is the main muscle activated for cough. The electrical stimulation (ES) is being widely used to fiber recruitment in many muscle groups and pathologies. The present study aimed to evaluate the efficiency of electrical stimulation with median frequencies (ETMF) in expiratory muscles strength and cough in patients with sequels of stroke. It is a quasi-experimental study (before and after) where 11 individuals were selected after stroke between 40 and 65 years, both sexes and hemodynamically stable. Patients were evaluated about clinic and respiratory scores (ventilometer, peak of expiratory flow (PEF) and manometer (MIP and MEP) and submitted to the protocol, which consisted of ETMF in expiratory muscle (abdominals rectus) with the unit of Russian current with $2,500 \mathrm{~Hz}$ carrier frequency modulated at $4 \mathrm{O} \mathrm{Hz}$ for 15 minutes by 15 sessions. In the initial assessment evidenced decreasing in inspiratory and expiratory muscular strength (MIP, MEP) and PEF when compared to predicted values in literature. In the end of ETMF it happened an increase in MIP, MEP without statistical significance ( $p=0.18$ and $p=0.29$ ) but the PEF has had an increase of $283.73 \mathrm{~L} /$ minute to $347.27 \mathrm{~L} /$ minute $(p=0.03)$. It can be seen that the ETMF was effective in the improvement of the parameters evaluated, with PEF being the greatest impact and statistical significance,

Estudo desenvolvido na Clínica Escola de Fisioterapia da Faculdade ASCES e UPS Casa Henrique - Caruaru (PE), Brasil.

'Graduando de Fisioterapia da Faculdade ASCES - Caruaru (PE), Brasil.

${ }^{2}$ Mestranda do Programa de Pós-graduação em Ciências Médicas pela Universidade Federal do Rio Grande do Sul (UFRGS) - Porto

Alegre (RS), Brasil.

${ }^{3}$ Fisioterapeuta pela Universidade Federal de Pernambuco (UFPE) - Recife (PE), Brasil.

${ }^{4}$ Doutorando em Nutrição pela UFPE - Cidade (PE), Brasil.

${ }^{5}$ Mestre em Fisiologia pela UFPE - Recife (PE), Brasil.

${ }^{6}$ Mestre em Pneumologia pela Universidade Federal de São Paulo (UNIFESP) - São Paulo (SP), Brasil. 
mais estudos com populações maiores se fazem necessários para análise dessa nova abordagem terapêutica.

Descritores I terapia por estimulação elétrica; acidente vascular cerebral; modalidades de fisioterapia; tosse. however further studies with larger populations are necessary to analyze this new therapeutic approach.

Keywords I electric stimulation therapy; stroke; physical therapy modalities; cough.

\section{INTRODUÇÃO}

O termo acidente vascular encefálico (AVE) é usado para definir o déficit neurológico (transitório ou definitivo) em uma área cerebral secundária a lesão vascular ${ }^{1}$ podendo ser de etiologia isquêmica ou hemorrágica ${ }^{2}$. De acordo com dados do DATASUS, no Brasil, até setembro de 2011, já houve 19.486 internações por AVE na rede pública, sendo 569 destes no estado de Pernambuco ${ }^{3}$.

Distúrbios respiratórios são complicações comuns em pacientes com AVE, ficando atrás apenas de complicações cardiovasculares entre as causas não neurológicas de piora após o quadro vascular cerebral ${ }^{1,4,5}$. Sabese que a lesão de córtex cerebral no AVE não causa alterações no drive respiratório ${ }^{6}$, no entanto, indivíduos hemiplégicos nos mais diversos graus, apresentam disfunção de musculatura ventilatória e consequentemente diminuição dos movimentos torácicos ${ }^{7}$. Essa alteração da mecânica pulmonar desencadeia diminuição na potência diafragmática e de musculatura respiratória, com bloqueio inspiratório prejudicando a função pulmonar, causando complicações respiratórias e internamentos hospitalares recorrentes ${ }^{8,9}$.

Os músculos abdominais atuam em sinergia com os eretores da coluna em coativação no segmento axial do tronco, para estabilizar e manter as posturas e tarefas dinâmicas voluntárias, além de ser o principal músculo utilizado na expiração forçada e tosse ${ }^{8,10}$. Estudos recentes relacionam a diminuição do trofismo e atividade muscular abdominal como uma possível sequela associada ao $\mathrm{AVE}^{11-13}$. Harraf et al. ${ }^{13}$ usando estimulação elétrica transcraniana encontraram alterações de pico de fluxo expiratório associadas à fraqueza da musculatura abdominal em pacientes com AVE, padrão que corrobora os trabalhos de outros autores ${ }^{9,11,12,14}$. As consequências diretas desse acometimento são a diminuição da pressão gerada durante a tosse levando a episódios de broncoaspiração e retenção de secreções em vias aéreas ${ }^{8,15}$.

Sabe-se que o treino de força no músculo reto abdominal através de dispositivos de expiração forçada, melhora o recrutamento de fibras, e consequentemente a tosse, mas existe uma série de condições clínicas e limitações que contraindica o uso destes para essa finalidade ${ }^{16-19}$. Além disso, já existem evidências que a eletroestimulação transcutânea em musculatura respiratória tem bons efeitos em pacientes crônicos, inclusive neurológicos, em quadros de atrofia muscular ${ }^{20-25}$. A aplicação da corrente Russa é feita com eletrodos de superfície na musculatura esquelética para fornecimento de corrente elétrica gerada por um estimulador que despolariza o neurônio motor e induz contrações musculares involuntárias, e já existem evidências científicas sobre sua aplicabilidade clínica ${ }^{26-28}$.

Ainda são escassos os estudos que associam a corrente Russa à melhora dos parâmetros respiratórios e a qualidade da tosse, com isso, nossa hipótese de trabalho é que esta modalidade de eletroestimulação pode aumentar o recrutamento e trofismo do músculo reto abdominal e com isso melhorar parâmetros pneumofuncionais em pacientes após AVE.

\section{METODOLOGIA}

Trata-se de um estudo quase-experimental (antes e depois) realizado na Clínica Escola de Fisioterapia da Faculdade ASCES e UPS Casa Henrique de Fisioterapia ambas situadas na cidade de Caruaru (PE) na qual foram recrutados, por conveniência, indivíduos portadores de sequelas de acidente vascular encefálico que encontravam-se em atendimento fisioterapêutico. O estudo foi aprovado pelo Comitê de Ética e Pesquisa da Faculdade ASCES, Protocolo no 119/10. Os participantes receberam informações sobre a pesquisa e assinaram o termo de consentimento livre e esclarecido.

Foram incluídos no estudo indivíduos entre 40 a 65 anos, ambos os sexos, estáveis hemodinamicamente e excluídos pacientes que já realizavam fisioterapia respiratória, tabagistas, incapacitados motor e cognitivamente para obedecer a comandos, pacientes com intolerância a corrente elétrica e qualquer problema de pele na região abdominal, cardiopatas, pacientes com câncer, gestantes, herniações abdominais e marcapasso cardíaco implantado. 
Os pacientes foram avaliados quanto aos dados pessoais (nome, idade, sexo, estado civil, entre outros), clínicos (presença de fatores de risco, patologias prévias ou coexistentes ao AVE), neurológicos (quanto ao diagnóstico neurológico, tipo de tônus, sequela neurológica, tipo de locomoção, e avaliação da sensibilidade) e avaliação pneumofuncional - através da coleta de sintomas respiratórios, sinais vitais, foi medida a força muscular respiratória pelas pressões respiratórias máximas, através da manovacuometria pelo manovacuômetro da DHD Healthcare Wampsville $^{\circledR}$, a velocidade com que o ar é expelido dos pulmões com o pico de fluxo expiratório através do aparelho Peak Flow meter Full Range da Personal Best ${ }^{\circledR}$ e observamos também o volume minuto, volume corrente, obtido pela divisão do volume minuto pela frequência respiratória, e a capacidade vital com o uso da ventilometria através do aparelho da Respirometer da Ferraris Respiratory ${ }^{\circledR}$.

Foi realizada uma avaliação inicial e logo após iniciou-se a intervenção através da eletroestimulação transcutânea de média frequência na musculatura expiratória (reto abdominal) com o aparelho de corrente russa Physiotonus Slin da BIOSET ${ }^{\circledR}$, com uma frequência portadora de $2.500 \mathrm{~Hz}$ modulada a $40 \mathrm{~Hz}$, trem de pulso de $350 \mu$ s, modo sincronizado, por 15 minutos, com média de frequência semanal de 2 vezes por semana, tempo de subida 3 segundos, tempo de sustentação de 4 segundos e tempo de descida 3, tempo $\mathrm{ON}$ 10 segundos tempo OFF 16 segundos por 15 sessões. Esse protocolo seguiu dados encontrados na literatura, respeitando o tipo de fibra muscular do reto abdominal (mista), a intensidade tolerada pelo paciente, e o tempo de terapia para evitar a fadiga ${ }^{25,29-31}$. Após a intervenção do protocolo, o grupo foi reavaliado quanto à condição respiratória geral (sinais vitais, presença de sintomas respiratórios, ausculta pulmonar e padrão respiratório) e parâmetros pneumofuncionais (ventilometria, pico de fluxo expiratório e manovacuometria).

Utilizou-se o programa estatístico SPSS (v. 12.0) para organização e tabulação dos dados. Após a verificação da normalidade da amostra pelo teste de ShapiroWilk, foi usado o teste $t$ de Student para comparação de dados antes e após a intervenção. Em todas as análises foi adotado o nível de significância de 5\% (p<0,05).

\section{RESULTADOS}

O presente estudo teve início com 15 pacientes, entretanto 4 pacientes foram excluídos da pesquisa pelo não comparecimento às sessões. As 15 sessões propostas foram terminadas por 11 pacientes, 63,7\% (7) eram do sexo masculino, 54,4\% (6) tiveram AVE do tipo hemorrágico, 72,8\% (8) da amostra apresentaram história de tabagismo e 81,8\% (9) eram portadores de hipertensão arterial. Quanto à mobilidade, quatro pacientes deambulavam de forma independente, seis utilizavam dispositivo auxiliar para marcha e apenas um paciente era cadeirante. $\mathrm{Na}$ Tabela 1 se encontram os resultados antropométricos e caracterização da amostra obtida na avaliação.

Quanto à avaliação pneumofuncional, o volume minuto, capacidade vital e volume corrente, não apresentaram alteração estatisticamente significante, tendo os valores $p>0,05$. A Tabela 2 mostra valores obtidos de pressões respiratórias máximas, pico de fluxo expiratório (PFE) e volume corrente expressos em \% previsto, que se encontravam abaixo dos valores previstos segundo Neder et al. ${ }^{32}$ para pressões respiratórias máximas obtidas, o PFE obtido também foi inferior aos valores propostos por Leiner et al. ${ }^{33}$, assim como os valores de volume corrente que se encontraram em níveis inferiores aos de normalidade de acordo com Schettino et al. $(2007)^{34}$.

$\mathrm{Na}$ Figura 1 podem-se observar as médias das pressões respiratórias e, na Figura 2, podem-se verificar as médias do PFE, antes e após a intervenção, respectivamente.

Os valores da pressão inspiratória máxima (Pimáx) e pressão expiratória máxima (Pemáx) após a intervenção obtiveram aumento, porém sem significância estatística considerável. Os valores de PFE apresentaram aumento estatisticamente significante após as sessões propostas.

Tabela 1. Caracterização da amostra estudada (media \pm desvio padrão)

$\begin{array}{lc} & \text { Avaliação inicial } \\ \text { Idade (meses) } & 54,6 \pm 7,8 \\ \text { Peso }(\mathrm{kg}) & 66,9 \pm 11,7 \\ \text { IMC }\left(\mathrm{kg} / \mathrm{m}^{2}\right) & 25,3 \pm 4,1 \\ \text { Tempo de AVE (meses) } & 38,4 \pm 44,4\end{array}$

IMC: índice de massa corporal; AVE: acidente vascular encefálico

Tabela 2. Valores absolutos previstos de pressões respiratórias máximas, pico de fluxo expiratório e volume corrente, expressos em \% do previsto pela literatura

$\begin{array}{lcc} & \text { Valores absolutos } & \text { \% previsto } \\ \text { PImáx }\left(\mathrm{CmH}_{2} \mathrm{O}\right) & 101,2 & 79,05 \\ \text { PEmáx }\left(\mathrm{CmH}_{2} \mathrm{O}\right) & 107,0 & 62,52^{*} \\ \text { PFE }(\mathrm{L} / \text { minuto }) & 481,5 & 58,56^{*} \\ \text { VC }(\mathrm{mL}) & 535,2 & 84,81\end{array}$

PImáx: pressão inspiratória máxima; PEmáx: pressão expiratória máxima; PFE: pico de fluxo expiratório; VC: volume corrente.

*diferença estaticamente significativa; PEmáx ( $p=0,03)$ e PFE $(p=0,00)$. 


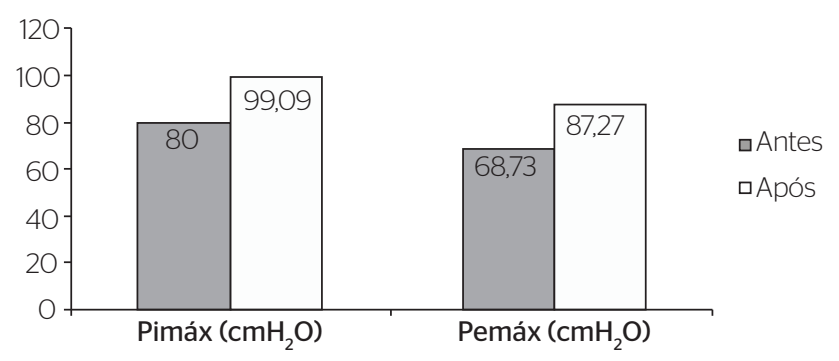

PImáx: pressão máxima inspiratória; PEmáx: pressão máxima expiratórias

Figura 1. Medidas das pressões máximas inspiratórias e expiratórias observadas antes e após as sessões propostas, (n=11)

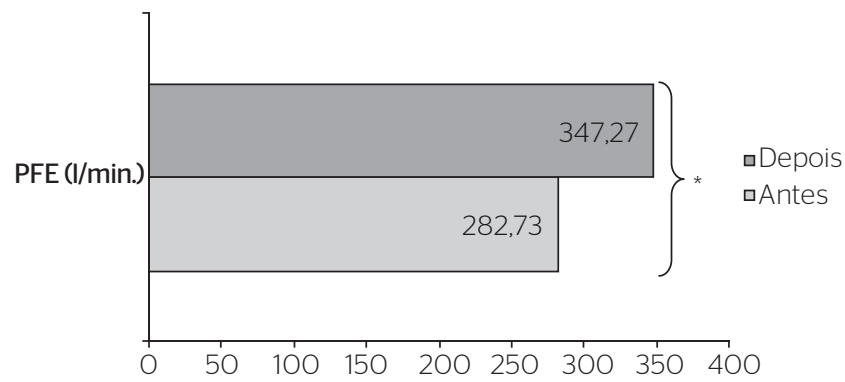

*diferença significativa ( $p=0,03)$; PFE: pico de fluxo expiratório

Figura 2. Medida do pico de fluxo expiratório antes e após as sessões propostas; $(n=11)$

\section{DISCUSSÃO}

A fisioterapia respiratória tem papel importante no processo de reabilitação do paciente após o AVE uma vez que, as alterações da biomecânica torácica, imobilidade relativa e diminuição do reflexo de tosse são comuns nesses indivíduos e parecem ter relação direta com infecções pulmonares ${ }^{5-7,35-37}$.

A literatura relata que a fraqueza do músculo reto abdominal é um padrão frequente em pacientes após o AVE e tem repercussão direta sobre parâmetros respiratórios ${ }^{8,11-14}$. A quantificação dessas repercussões também já foi descrita em estudos com pacientes hemiparéticos, sendo a PEmáx e PFE medidas confiáveis na análise da força expiratória e capacidade de geração de fluxo expiratório ${ }^{9,14,32,38,39}$. Essas medidas foram utilizadas nesse estudo, e ambas apresentaram diminuição quanto aos valores de normalidade preditos, com significância estatística, o que corrobora a literatura, como expresso na Tabela 2. Meneghetti et al. ${ }^{9}$ relataram diminuição da Pimáx e Pemáx em um grupo de 22 pacientes com faixa etária variável entre 30 e 87 anos pós-AVE. Teixeira-Salmela et al. ${ }^{12}$ também obtiveram achados semelhantes comparando grupo de 16 pacientes pós-AVE com um grupo controle composto por 19 pessoas saudáveis. Ward et al. ${ }^{14}$ observaram diminuição do $\mathrm{PFE}$ em
18 indivíduos pós-AVE em relação ao controle com mesma média de idade.

A corrente russa foi contemplada nesse estudo por ser uma modalidade de eletroestimulação de média frequência $(2.500 \mathrm{~Hz})$ com alta tolerabilidade e capacidade de recrutamento de várias unidades motoras de forma sincrônica, gerando força e hipertrofia muscular ${ }^{26-28}$. A frequência de $40 \mathrm{~Hz}$ foi escolhida pelas características da fibra (mista) do músculo reto abdominal ${ }^{40,41} \mathrm{e}$ a duração de pulso pelo tamanho, sendo protocolado para músculos longos uma faixa entre 300 e $400 \mu$ s, a utilizada foi de $350 \mu \mathrm{s}^{7,20,21,25,42}$. Os tempos on e off foram determinados de maneira que houvesse recrutamento muscular por um período efetivo com uma margem de segurança, afim de evitar a fadiga, ficando 10 segundos on e 16 off $^{43}$.

A eletroestimulação é uma alternativa recente na reabilitação pulmonar. Langer et al ${ }^{44}$ relatam o uso da eletroestimulação na melhora da força muscular e capacidade de exercício em pacientes com doença pulmonar obstrutiva crônica (DPOC) em musculatura apendicular. Outros autores encontraram resultados semelhantes nesta população, mas estes estudos tinham como objetivo a melhora de parâmetros cardiovasculares, através do fortalecimento de musculatura distal e não da tosse especificamente ${ }^{21,25,27}$. Lee et al. ${ }^{23}$ observaram através da eletroestimulação abdominal um incremento da força muscular expiratória em um paciente portador de lesão medular. Não há relatos na literatura sobre outros estudos que utilizaram a corrente russa para finalidades pneumofuncionais em pacientes com AVE.

Os achados verificados no presente estudo apontam que a eletroestimulação parece ser eficiente na promoção de melhora da capacidade de tosse, mensurado através do aumento da PFE. O estudo teve limitações quanto ao número de participantes devido à presença de condições clínicas e físicas nos pacientes recrutados inicialmente, não sendo possível com isso, a avaliação das pressões inspiratórias e expiratórias e uso da eletroterapia com segurança. Atribuiu-se a esse perfil de amostra limitada o fato dos dados de aumento do PEmáx não serem expressivos estaticamente.

\section{CONCLUSÃO}

Os pacientes avaliados apresentaram diminuição significativa da força muscular expiratória e do pico de fluxo expiratório na avaliação inicial, comparados com valores de normalidade previstos pela literatura 
A eletroestimulação transcutânea de média frequência parece ter eficácia no incremento do PFE, tal incremento é vital para o que o paciente obtenha uma tosse eficaz, prevenindo possíveis infecções respiratórias.

A literatura sobre o tema ainda é bastante escassa, fazendo-se necessária a realização de pesquisas com maior número de participantes, para avaliar a eficácia dessa nova abordagem.

\section{REFERÊNCIAS}

1. Radanovic M. Características do atendimento de pacientes com acidente vascular cerebral em hospital secundário. Arq NeuroPsiquiatr. 2000;58(1):99-106.

2. Remesso GC, Chiappetta ALML, Aguiar AS, Fukujima MM, Prado GF. Swallowing disorders after ischemic stroke. Arq Neuro-Psiquiatr. 2011;69(5):785-9.

3. DATASUS. Morbidade Hospitalar do SUS - por local de residência. Ministério da Saúde do Brasil [cited 2012 Dec 17]. Available from: http://tabnet.datasus.gov.br/cgi/tabcgi.exe?sih/cnv/nrrs.def

4. Lan MY, Wu SJ, Chang YY, Chen WH, Lai SL, Liu JS. Neurologic and non-neurologic predictors of mortality in ischemic stroke patients admitted to the intensive care unit. J Formos Med Assoc. 2006;105(8):653-8.

5. Kuys S, Brauer S, Ada L. Routine physiotherapy does not induce a cardiorespiratory training effect poststroke, regardless of walking ability. Physiother Res Int 2006;11(4):219-27.

6. Lanini B, Bianchi R, Romagnoli I, Coli C, Binazzi B, Gigliotti F, et al. Chest wall kinematics in patients with hemiplegia. Am J Respir Crit Care Med. 2003;168(1):109-13.

7. Billinger SA, Coughenour E, Mackay-Lyons MJ, Ivey FM. Reduced cardiorespiratory fitness after stroke: biological consequences and exercise-induced adaptations. Stroke Res Treat. 2012;2012:959120.

8. Pompeu SMAA, Pompeu JE, Rosa M, Silva MR. Correlação entre função motora, equilíbrio e força respiratória pós Acidente Vascular Cerebral. Rev Neurocienc. 2011:19(4):614-20.

9. Meneghetti CHZ, Figueiredo VE, Guedes CAV, Batistela ACT. Avaliação da força muscular respiratória em indivíduos acometidos por acidente vascular cerebral. Rev Neurocienc 2011;19(1):56-60.

10. Julia PE, Sa'ari MY, Hasnan N. Benefit of triple-strap abdominal binder on voluntary cough in patients with spinal cord injury. Spinal Cord. 2011:49(11):1138-42.

11. Marcucci FCl, Cardoso NS, Berteli KdS, Garanhani MR, Cardoso JR. Alterações eletromiográficas dos músculos do tronco de pacientes com hemiparesia após acidente vascular encefálico. Arq NeuroPsiquiatr. 2007;65(3b):900-5.

12. Teixeira-Salmela LF, Parreira VF, Britto RR, Brant TC, Inácio ÉP, Alcântara TO, et al. Respiratory pressures and thoracoabdominal motion in community-dwelling chronic stroke survivors. Arch Phys Med Rehab. 2005;86(10):1974-8.

13. Harraf F, Ward K, Man W, Rafferty G, Mills K, Polkey M, et al. Transcranial magnetic stimulation study of expiratory muscle weakness in acute ischemic stroke. Neurology 2008;71(24):2000-7.
14. Ward K, Seymour J, Steier J, Jolley CJ, Polkey MI, Kalra L, et al. Acute ischaemic hemispheric stroke is associated with impairment of reflex in addition to voluntary cough. Eur Respir J. 2010;36(6):1383-90.

15. Widdicombe JG, Addington WR, Fontana GA, Stephens RE. Voluntary and reflex cough and the expiration reflex; implications for aspiration after stroke. Pulm Pharmacol Ther. 2011;24(3):312-7.

16. Ishida H, Hirose R, Watanabe S. Comparison of changes in the contraction of the lateral abdominal muscles between the abdominal drawing-in maneuver and breathe held at the maximum expiratory level. Man Ther. 2012;17(5):427-31.

17. Matsumura T, Saito T, Fujimura H, Shinno S, Sakoda S. Lung inflation training using a positive end-expiratory pressure valve in neuromuscular disorders. Intern Med. 2012;51(7):711-6.

18. Dohna-Schwake C, Ragette R, Teschler H, Voit T, Mellies U. IPPBassisted coughing in neuromuscular disorders. Pediatr Pulmonol. 2006:41(6):551-7.

19. Kim K, Fell D, Lee JH. Feedback Respiratory Training to Enhance Chest. Expansion and Pulmonary Function in Chronic. Stroke: A Double-Blind, Randomized Controlled. J Phys Ther Sci. 2011;23(1):74-9.

2O. Gerovasili V, Stefanidis K, Vitzilaios K, Karatzanos E, Politis P, Koroneos A, et al. Electrical muscle stimulation preserves the muscle mass of critically ill patients: a randomized study. Criti Care. 2009;13(5):R161.

21. Gerovasili V, Tripodaki E, Karatzanos E, Pitsolis T, Markaki V, Zervakis $D$, et al. Short-term systemic effect of electrical muscle stimulation in critically ill patients. Chest. 2009;136(5):1249-56.

22. Borges VM, Oliveira LRC, Peixoto E, Carvalho NAA. Fisioterapia motora em pacientes adultos em terapia intensiva. Rev Bras Ter Intensiva. 2009;21(4):446-52.

23. Lee BB, Boswell-Ruys C, Butler JE, Gandevia SC. Surface functional electrical stimulation of the abdominal muscles to enhance cough and assist tracheostomy decannulation after high-level spinal cord injury. J Spinal Cord Med. 2008;31(1):78-82.

24. Gallas S, Marie JP, Leroi AM, Verin E. Sensory Transcutaneous electrical stimulation improves post-stroke dysphagic patients. dysphagia. 2010;25(4):291-7.

25. Vivodtzev I, Lacasse Y, Maltais F. Neuromuscular electrical stimulation of the lower limbs in patients with chronic obstructive pulmonary disease. J Cardiopulm Rehabil Prev. 2008;28(2):79-91.

26. Abdalla DR, Bertoncello D, Carvalho LC. Avaliação das propriedades mecânicas do músculo gastrocnêmio de ratas imobilizado e submetido à corrente russa. Fisioter Pesq. 2009:16(1):59-64.

27. Araúijo JM, Santos E. Dois protocolos distintos de reabilitação pulmonar em pacientes portadores de doença pulmonar obstrutiva crônica. Relato de casos e revisão de literatura. Rev Bras Clin Med. 2012:10(1):87-90

28. Avila MA, Brasileiro JS, Salvini TF. Electrical stimulation and isokinetic training: effects on strength and neuromuscular properties of healthy young adults. Rev Bras Fisioter. 2008;12(6):435-40.

29. Martins AD. Análise eletromiográfica do musculo reto abdominal pré e pós aplicação de um portocolo de corrente russa associada ou não a exercícios abdominais em mulheres sedentárias [dissertação]. Criciúma: Universidade do Extremo Sul de Santa Catarina, 2009.

30. Briel AF, Massoni J. Efeito da eletroestimulação por corrente russa e exercício de fortalecimento da força e trofismo muscular do quadriceps femoral não dominante. Revista de Biologia e Saúde da UNISEP. 2009;3(1):34-45. 
31. Boitano LJ. Management of airway clearance in neuromuscular disease. Respir Care. 2006;51(8):913-22.

32. Neder JA, Andreoni S, Lerario MC, Nery LE. Reference values for lung function tests. II. Maximal respiratory pressures and voluntary ventilation. Braz J Med Biol Res. 1999;32:719-27.

33. Leiner GC, AbramowitzS, Small MJ, Stenby VB, Lewis WA. Useasa Clinical Test of Ventilatory Function. Am Rev Respir Dis. 1963;88(6):644-51.

34. Schettino GPP, Reis MAS, Galas F, Park M, Franca S, Okamoto V. III Consenso Brasileiro de Ventilação Mecânica - ventilação não invasiva com pressão positiva. J Bras Pneumol. 2007;33(Suppl 2):S92-105.

35. Olai L, Borgquist L, Svärdsudd K. Health problems in elderly patients during the first post-stroke year. Ups J Med Sci. 2012; 117(3):318-27.

36. Zhou Z, Vincent F, Salle JY, Antonini MT, Aliamus V, Daviet JC. Acute stroke phase voluntary cough and correlation with maximum phonation time. Am J Phys Med Rehabil. 2012;91(6):494-500.

37. Hug A, Mürle B, Dalpke A, Zorn M, Liesz A, Veltkamp R. Usefulness of serum procalcitonin levels for the early diagnosis of stroke-associated respiratory tract infections. Neurocrit Care. 2011;14(3):416-22.
38. Costa D, Gonçalves HA, Lima LP, Ike D, Cancelliero KM, Montebelo MIL. Novos valores de referência para pressões respiratórias máximas na população brasileira. J Bras Pneumol. 2010;36(3):306-12.

39. Parreira VF, França DC, Zampa CC, Fonseca MM, Tomich GM, Britto RR. Pressões respiratórias máximas: valores encontrados e preditos em indivíduos saudáveis. Rev Bras Fisioter. 2007:11(5):361-8.

40. Marchetti PH. Investigações sobre o controle neuromotor do músculo reto do abdome [dissertação]. São Paulo: Universidade de São Paulo, 2005.

41. Häggmark T, Thorstensson A. Fibre types in human abdominal muscles. Acta Physiol Scand. 1979;107(4):319-25.

42. Vanderthommen M, Crielaard JM. Muscle electric stimulation in sports medicine. Rev Med Liege. 2001;56(5):391-5.

43. Brasileiro JS, Castro CES, Parizotto NA. Parâmetros manipuláveis Clinicamente na Estimulação Elétrica Neuromuscular (EENM). Fisioter Bras. 2002;3(1):16-24.

44. Langer D, Probst VS, Pitta F, Burtin C, Hendriks E, Schan CPVD, et al. Guia para prática clínica: Fisioterapia em Pacientes com Doença Obstrutiva Crônica (DPOC). Rev Bras Fisioter. 2009;13(3):183-204. 